\title{
Real-time assessment of inflammation and treatment response in a mouse model of allergic airway inflammation
}

\author{
Virna Cortez-Retamozo,, ${ }^{1,2}$ Filip K. Swirski, ${ }^{2}$ Peter Waterman,, 1,2 \\ Hushan Yuan, ${ }^{2}$ Jose Luiz Figueiredo,, ${ }^{1,2}$ Andita P. Newton,, ${ }^{1,2}$ Rabi Upadhyay, ${ }^{2}$ \\ Claudio Vinegoni, ${ }^{1}$ Rainer Kohler, ${ }^{2}$ Joseph Blois, ${ }^{2}$ Adam Smith, ${ }^{2}$ Matthias Nahrendorf, ${ }^{1,2}$ \\ Lee Josephson, ${ }^{2}$ Ralph Weissleder, ${ }^{1,2}$ and Mikael J. Pittet ${ }^{1,2}$
}

${ }^{1}$ Center for Systems Biology and ${ }^{2}$ Center for Molecular Imaging Research,

Massachusetts General Hospital and Harvard Medical School, Boston, Massachusetts, USA.

\begin{abstract}
Eosinophils are multifunctional leukocytes that degrade and remodel tissue extracellular matrix through production of proteolytic enzymes, release of proinflammatory factors to initiate and propagate inflammatory responses, and direct activation of mucus secretion and smooth muscle cell constriction. Thus, eosinophils are central effector cells during allergic airway inflammation and an important clinical therapeutic target. Here we describe the use of an injectable MMP-targeted optical sensor that specifically and quantitatively resolves eosinophil activity in the lungs of mice with experimental allergic airway inflammation. Through the use of real-time molecular imaging methods, we report the visualization of eosinophil responses in vivo and at different scales. Eosinophil responses were seen at single-cell resolution in conducting airways using nearinfrared fluorescence fiberoptic bronchoscopy, in lung parenchyma using intravital microscopy, and in the whole body using fluorescence-mediated molecular tomography. Using these real-time imaging methods, we confirmed the immunosuppressive effects of the glucocorticoid drug dexamethasone in the mouse model of allergic airway inflammation and identified a viridin-derived prodrug that potently inhibited the accumulation and enzyme activity of eosinophils in the lungs. The combination of sensitive enzyme-targeted sensors with noninvasive molecular imaging approaches permitted evaluation of airway inflammation severity and was used as a model to rapidly screen for new drug effects. Both fluorescence-mediated tomography and fiberoptic bronchoscopy techniques have the potential to be translated into the clinic.
\end{abstract}

\section{Introduction}

Asthma is a chronic inflammatory disease of the airways characterized by mucus secretion, bronchial hyperresponsiveness, and variable and reversible airflow obstruction. The disease affects approximately 300 million people worldwide and is continuously increasing in prevalence. Airway inflammation, a central component of most manifestations of asthma, results from a reaction to an inhaled environmental substance (e.g., an allergen) that triggers a series of inflammatory events (1-4). In response to inhalation of such agents, airways of sensitized individuals release eotaxin $(5,6)$ and leukotrienes $(7)$ that rapidly recruit eosinophils. Eosinophils contribute decisively to the pathology of asthma because they produce proteinases that degrade and remodel tissue extracellular matrix; secrete various proinflammatory factors that promote the recruitment, survival, and activation of other immune effector cells; and promote mucus secretion and smooth muscle cell constriction $(8,9)$. Several recent studies indicate that eosinophils also elicit the expression of cytokines that activate and recruit deleterious Th2 lymphocytes to the lung $(8,10-12)$. Thus the pervading data imply that

Nonstandard abbreviations used: alum, aluminum hydroxide; BAL, bronchoalveolar lavage; FMT, fluorescence-mediated molecular tomography; FRI, fluorescence reflectance imaging; IVM, intravital microscopy, NIRF, near-infrared fluorescence.

Conflict of interest: Peter Waterman and Ralph Weissleder are consultants for VisEn Medical.

Citation for this article: J. Clin. Invest. doi:10.1172/JCI36335 eosinophils are the primary cellular mediators of the asthmatic response to allergen exposure.

Over the last century, research on the inflammatory components that cause the clinical features of asthma has moved from invasive (postmortem) histological studies (13) to less invasive approaches, such as withdrawal of bronchoalveolar lavage (BAL) fluids or bronchial biopsies (14-16). Noninvasive anatomical techniques, such as spirometry (17), X-ray CT (18), MRI $(19,20)$, and PET (21), have also been developed to measure ventilation, perfusion, and alveolar-capillary gas transfer. However, there is a need for more efforts to take advantage of state-of-the-art imaging methods and to develop and validate diseased lung-specific biomarkers as valuable adjuncts to diagnosis and assessment of treatment response (22). Molecular imaging techniques for the study of allergic airway inflammation are not yet available. The reasons to develop and validate such tools are 2 -fold. First, in vivo molecular imaging may improve our fundamental understanding of immune responses because the behavior of immune cells in tissues is dictated by local factors that often cannot be reproduced in vitro (23). Second, clinically applicable molecular imaging techniques may improve our ability to diagnose and stage disease and to assess treatment efficacy. Indeed, current approaches to assessing molecular endpoints in asthma require tissue and blood sampling and, in animal models, sacrifice at defined time points. Thus, immune pathways that are "turned on" during allergic airway inflammation may serve as targets for in vivo molecular imaging of disease progression. 
A
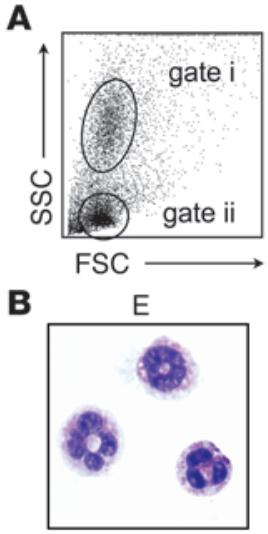

C $E$

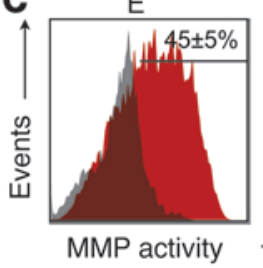

D

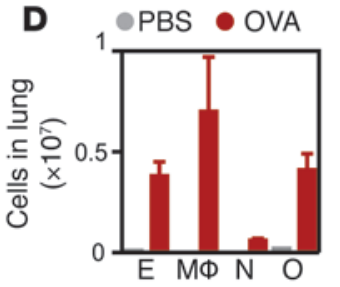

Gate i

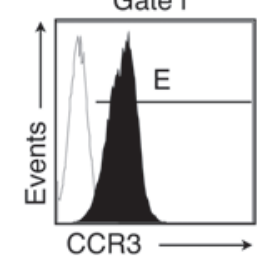

$M \Phi$

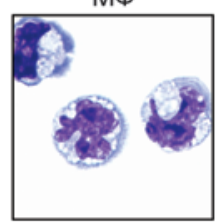

$M \Phi$

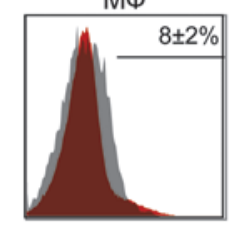

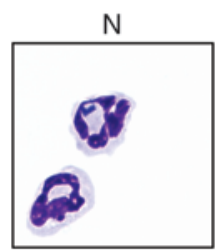

$\mathrm{N}$

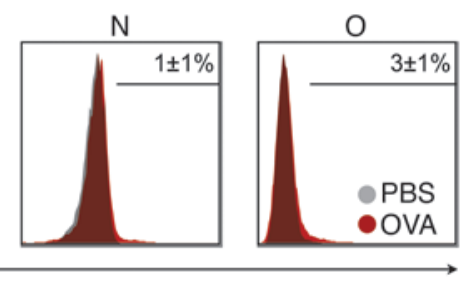

$\mathrm{O}$
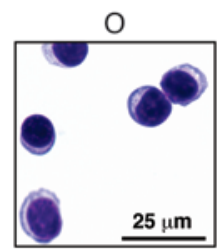
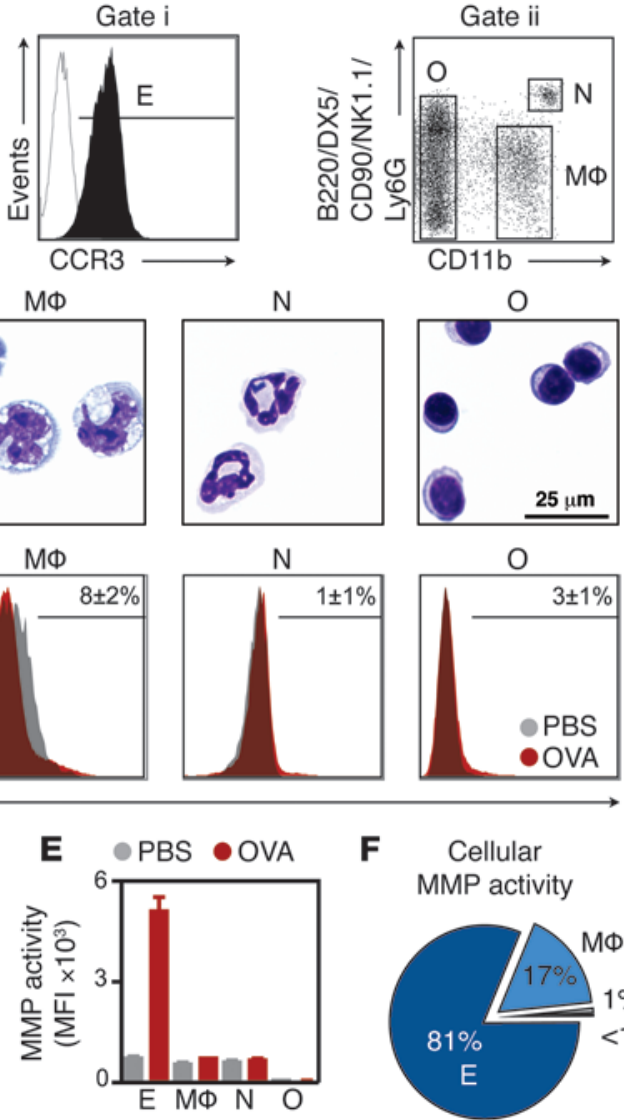

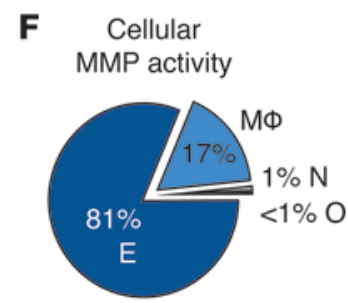

\section{Figure 1}

Eosinophils in inflamed lungs display potent MMP activity. E, eosinophils; M $\phi$, monocytes/macrophages; $\mathrm{N}$, neutrophils, $\mathrm{O}$, other cells. (A) Identification of cell populations in single-cell suspensions of digested lungs from OVA-treated and control PBS-treated mice. FSC, forward scatter; SSC, side scatter. (B) $\mathrm{H} \& \mathrm{E}$ staining of flow-sorted cells obtained from digested lung tissue and identified based on expression of specific cell surface markers. Scale bar: $25 \mu \mathrm{m}$. (C) Flow cytometric analysis revealed increased MMP activity in eosinophils in inflamed lungs. Numbers denote percent MMP-positive cells in inflamed versus control lung. (D) Total cell counts in lungs. (E) MMP mean fluorescence intensity (MFI) in each cell type in lung, as identified by flow cytometry. (F) Contribution of cell types to MMP activity in inflamed lungs based on results in $\mathbf{D}$ and $\mathbf{E}$. Data are representative of at least 3 independent experiments, with $n=3-5$ per experiment and per group. Data are mean \pm SEM.
Pulmonary eosinophils and other cells, such as macrophages, mast cells, and smooth muscle cells, produce MMPs - such as MMP-2, - 3, -9, -12, -13, and $-14-$ as well as cathepsins B, S, L, $\mathrm{H}$, and $\mathrm{K}$, which are thought to contribute to the pathogenesis of asthma (24-36). MMPs have received heightened attention because they are elevated in experimental models of allergic airway inflammation $(24-29,36)$ and in the airways of asthmatics $(25$, 33-36). They also degrade tissue extracellular matrix; promote the recruitment, proliferation, and survival of inflammatory cells; and participate directly in bronchoconstriction and airway remodeling $(25,37,38)$. To varying degrees, the biological activities mentioned above are found even in patients with mild asthmatic symptoms (39), which suggests that inflammation is an underlying pathologic feature in symptomatic patients.

Here we hypothesized that noninvasive molecular imaging of eosinophil-associated proteinase activity may inform on disease severity. To test this hypothesis, we combined the use of specific enzyme-targeted optical sensors with different molecular imaging modalities and performed noninvasive and real-time investigations of lung parenchyma and conducting airways in a mouse model of allergic airway inflammation. We aimed to define which enzymes may serve as sensitive biomarkers of inflammation and whether these biomarkers could be used to diagnose the modulation of inflammation upon therapeutic intervention. We report that a pan-MMP sensor informed noninvasively on extent and quality of allergic airway inflammation. Eosinophil-mediated MMP activity was visualized by intravital microscopy (IVM), fluorescence-mediated molecular tomography (FMT), and near-infrared fluorescence (NIRF) fiberoptic bronchoscopy.

\section{Results}

Eosinophils display potent MMP activity in inflamed lungs. Initially, we tested whether eosinophils exhibit distinct enzyme activities that can be detected with molecular sensors (40). We used pan-MMPand pan-cathepsin-targeted optical reporters that consist of 3 building blocks: near-infrared fluorochromes, specific protease peptide substrates, and partially methoxypegylated graft copolymers. Fluorochromes are initially quenched, but cleavage of the scaffold by specific proteinases releases fluorochromes and generates extensive fluorescence. Each active moiety of enzyme can activate multiple reporters, leading to signal amplification. Here we administered MMP and cathepsin sensors in mice with inflamed or resting lungs and compared the enzyme activities mediated by eosinophils and other cells (e.g., macrophages, neutrophils, and lymphocytes). This involved sensitization of female BALB/c mice with chicken egg OVA in the presence of aluminum hydroxide (alum), followed by challenge with OVA and i.v. injection of MMPand cathepsin-targeted sensors (Supplemental Figure 1; supplemental material available online with this article; doi:10.1172/ JCI36335DS1). At 1 day after the last antigen challenge, the lung accumulated at least 4 types of leukocytes, which we identified by flow cytometry as eosinophils (SSC ${ }^{\text {hi }}$ CCR $3^{\text {hi }}$ ), monocytes/macrophages $\left(\mathrm{CD} 11 \mathrm{~b}^{\text {hi }} \mathrm{B} 220^{\mathrm{lo}} \mathrm{DX} 5^{\mathrm{lo}} \mathrm{CD} 90^{\mathrm{lo}} \mathrm{NK} 1.1^{\mathrm{lo}} \mathrm{Ly} 6 \mathrm{G}^{\mathrm{lo}}\right)$, neutrophils (CD11b ${ }^{\text {hi }}$ 220 ${ }^{\text {hi DX }} 5^{\text {hi }}$ CD90hiNK1.1 hi Ly6G hi), and other cells (CD11b $b^{\text {lo }}$, mostly lymphocytes; Figure $\left.1 \mathrm{~A}\right)$. Microscopic examination of flow-sorted cell subsets confirmed their morphologic features (Figure 1B). Coregistration of proteinase activity in each cell type identified eosinophils as the primary source of MMP activity in inflamed lungs (Figure 1C). Cathepsin activity also increased in 
A

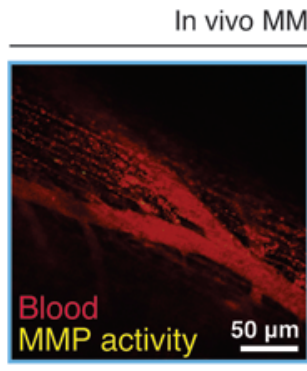

Sens $\times 2$

Chall -

C

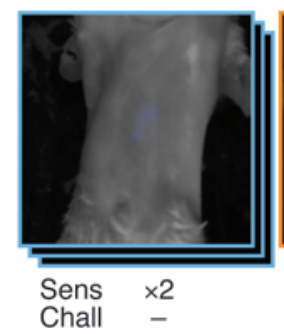

E

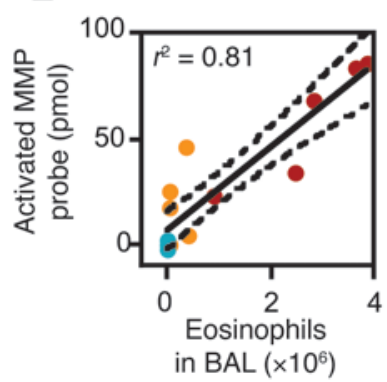

activity (IVM)

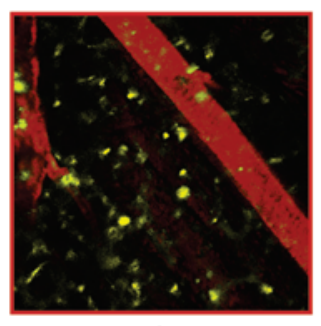

$\times 2$

$+$

In vivo MMP activity (FMT)
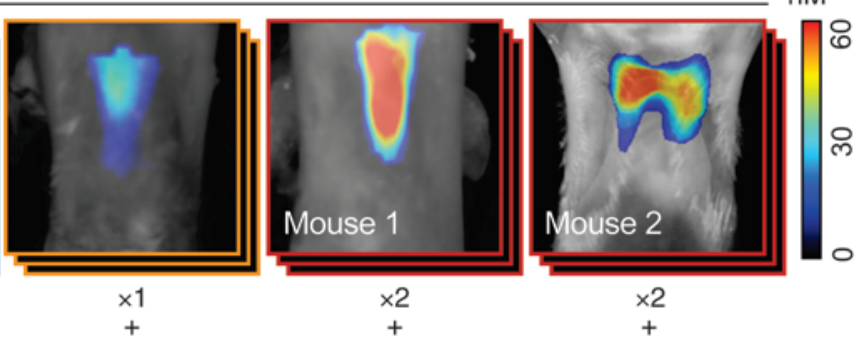

$\mathrm{nM}$

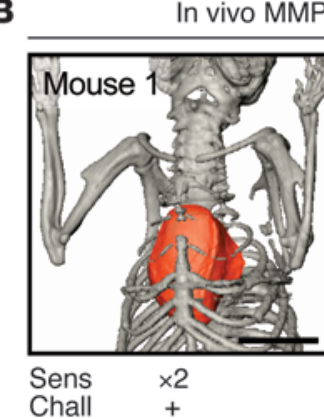

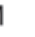

F

Ex vivo MMP activity (FRI)
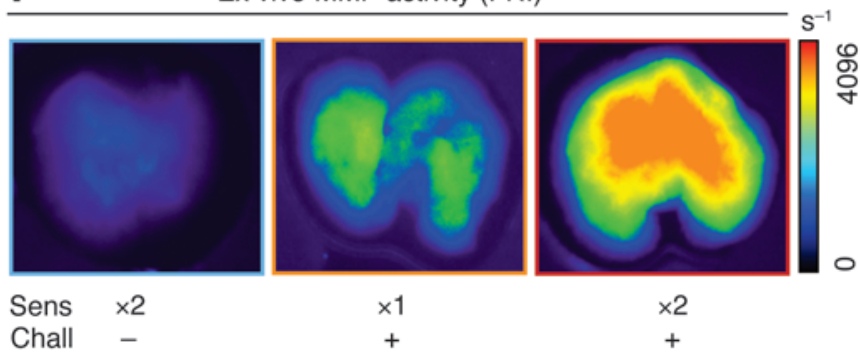

$\mathbf{G}$

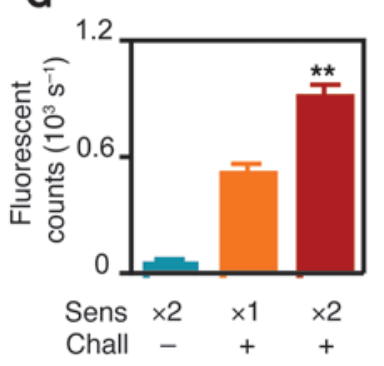

Figure 2

In vivo detection of eosinophil-mediated MMP activity correlates with disease severity. Sens, sensitization $(\times 1$, once; $\times 2$, twice); Chall, challenge with OVA. (A) IVM showed single-cell MMP activity in inflamed versus control lungs. (B) Virtual 3-dimensional rendering of MMP activity in inflamed lungs of 2 different mice and acquired by FMT-CT. MMP activity localized centrally to the lower trachea and major bronchi in mouse 1 , and to the right and left lobes of the lung in mouse 2. (C and D) FMT measurement of MMP activity in whole lungs of mice with no (unchallenged; blue), moderate (sensitized once with OVA/alum; orange), or potent (sensitized twice with OVA/alum; red) airway inflammation. Mice 1 and 2 from $B$ are shown. (E) MMP activity in vivo positively correlated with the number of eosinophils in BAL fluids. (F and $\mathbf{G}) \mathrm{FRI}$ of excised lungs confirmed the FMT results. At least 3 independent experiments were performed, with $n=3-5$ per experiment and per group. Data are mean \pm SEM. ${ }^{*} P<0.05,{ }^{* *} P<0.01$ versus once-sensitized group. Scale bars: $50 \mu \mathrm{m}(\mathbf{A}) ; 1 \mathrm{~cm}(\mathbf{B})$.

activated eosinophils, albeit to a lower degree (data not shown). Taking into account the abundance of each cell type in inflamed lungs (Figure 1D) and the enzyme activity of single cells (Figure $1 \mathrm{E}$ ), we found that eosinophils contributed to greater than $80 \%$ of pulmonary MMP activity in inflamed lungs (Figure 1F). Thus, imaging of MMP activity should inform on eosinophil-associated responses during allergic airway inflammation.

In vivo detection of eosinophil-mediated MMP activity correlates with disease severity. We used different in vivo imaging approaches to resolve eosinophil-associated MMP activity at microscopic and macroscopic resolutions in mice previously administered with the MMP-targeted sensor. Microscopic analysis at subcellular resolution involved IVM of lung parenchyma using ultrathin stick objectives and keyhole surgical access (41). The approach allowed signal detection up to $200 \mu \mathrm{m}$ in depth and revealed pan-MMP activity in inflamed, but not resting, lungs (Figure 2A). Nearly all MMP activity originated from single cells that had been identified as tissue eosinophils. This finding suggests that active enzymes exert their activity extensively within or on the cell membrane of eosinophils, with limited activity in the extracellular space. We also used FMT (Supplemental Figure 2) for quantitative reconstructions of fluorochrome concentration in 3 dimensions and at submillimiter resolution through the entire mouse. Typical monitoring readout during isoflurane anesthesia, which was used during FMT imaging for mouse immobilization, showed that inspiration comprised only $10 \%-20 \%$ of the respiratory cycle (downward slope on pressure-time curve; Supplemental Figure 3). During inspiration, the chest wall moves out and the diaphragm caudally by about $1-2 \mathrm{~mm}$. Because imaging was performed continually, the acquisition of fluorescent signal was heavily weighted toward the expiratory/inactive state. Although there may be some degree of partial volume effects or motion-induced artifacts, this movement was in the range of the 
A

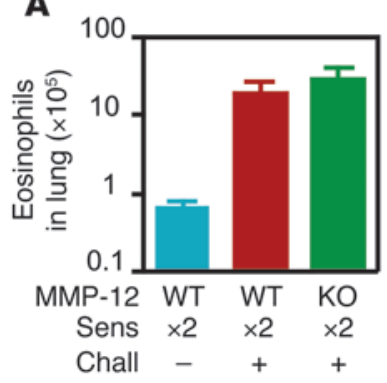

c

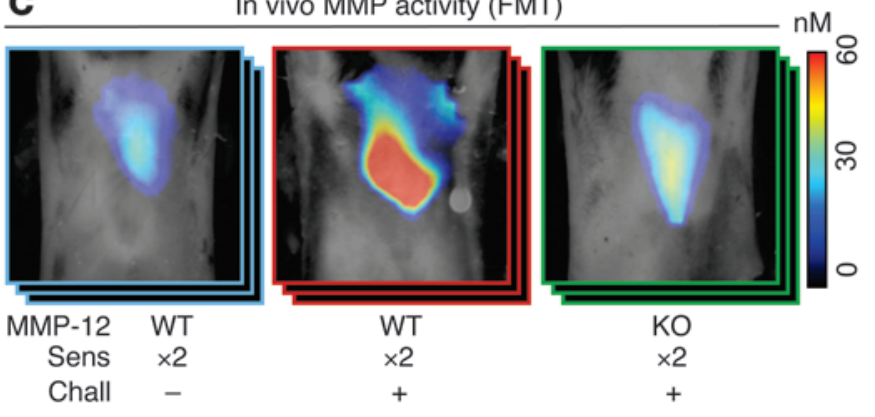

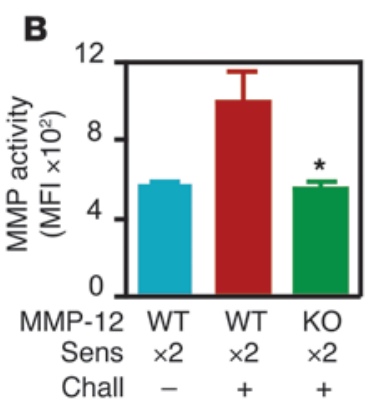

D

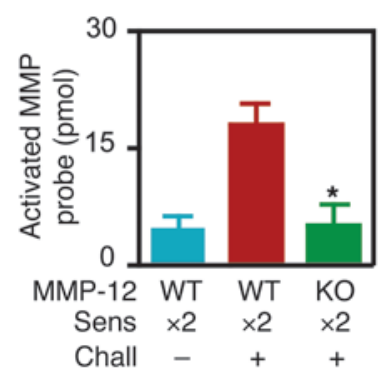

\section{Figure 3}

Decreased eosinophil-mediated MMP activity in MMP-12-deficient mice. (A) MMP-12-deficient (KO, green) and wild-type mice (red) recruited eosinophils to the inflamed lung. Unchallenged mice (blue) served as controls. (B) MMP-12-deficient mice showed decreased MMP activity in lung eosinophils, as identified by flow cytometry. (C and D) FMT imaging revealed decreased MMP activity in inflamed lungs of MMP-12deficient mice. At least 2 independent experiments were performed, with $n=3$ per experiment and per group. Data are mean \pm SEM. ${ }^{*} P<0.05$ versus OVA-challenged WT. spatial resolution of the FMT system. Furthermore, volumes of interest were chosen in a way that avoided the outermost $2 \mathrm{~mm}$ of the lung, thereby excluding areas with potential motion-related partial volume effects for quantification. We fused FMT reconstructions with high-resolution X-ray CT (FMT-CT) to improve image visualization (Figure 2B, Supplemental Figure 2C, and Supplemental Video 1) and identified that MMP activity in mice challenged with OVA localized either centrally to the lower trachea and major bronchi or to the right and left lobes of the lung. Central MMP activity is likely the result of the route of administration for the allergen (OVA challenges were performed i.n.) and the subsequent recruitment of eosinophils, the main cellular source of MMP activity, in the mucosa of the conducting airways. Previous studies have also reported the presence of inflammatory cell populations within the conducting airway mucosa (42). Quantitative FMT analysis of enzyme activity in lung parenchyma of different cohorts of mice revealed significantly increased eosinophil-associated MMP activity in animals with more severe allergic inflammation (Figure 2, C and D, Supplemental Figures 1 and 2, and Supplemental Videos 2-4). The registered enzyme activity positively correlated with eosinophilic infiltration (Figure 2E) and with IL-5 concentration (data not shown) in the corresponding BAL fluids. Excised lungs analyzed by fluorescence reflectance imaging (FRI) confirmed our in vivo observations (Figure 2, F and G). These data indicate that in vivo activity of the MMP-targeted optical reporter can serve as a surrogate for the eosinophilic response occurring in the airways.

We coregistered MMP and cathepsin activities at $680 \mathrm{~nm}$ and $750 \mathrm{~nm}$, respectively, during the in vivo FMT imaging procedures. In agreement with flow cytometry data, we found slight differences of cathepsin activity in lungs with moderate or potent allergic airway inflammation (Supplemental Figure 4), yet this differential activity was not significant and did not correlate with eosinophilic infiltration in the BAL. Thus, cathepsin activity failed to inform on the severity of the allergic inflammatory response.

Activation of the MMP-targeted optical sensor in vivo. Proteinases produced by eosinophils include various MMPs, some of which have been involved in the pathology of asthma (36). A systemslevel approach integrating a gene expression compendium of microarray data sets from multiple sources identified MMP-12 as the only MMP that was persistently upregulated in lungs upon induction of OVA-derived allergic airway inflammation (43). MMP12 may be a central enzyme produced by eosinophils because it is also expressed at high levels in patients with airway inflammation $(33,44,45)$. In addition, it degrades extracellular matrix components, promotes cell migration and remodeling, and orchestrates the production of IL-13 in mouse models of airway inflammation $(24,26,31)$. Thus, we thought to test whether the MMP sensor is activated in mice deficient in MMP-12. We prepared single-cell suspensions from lung tissue of wild-type and MMP-12-deficient animals previously sensitized and challenged with OVA and found that eosinophils accumulated efficiently in the lungs of animals in both groups (Figure 3A) but showed compromised MMP activity on a per-cell basis in MMP-12-deficient mice (Figure 3B). Noninvasive FMT analysis also revealed massive reduction of enzyme activity in the lungs of MMP-12-deficient mice (Figure 3, C and D), which was further confirmed ex vivo by FRI on excised lung tissue (Supplemental Figure 5, A and B). These results indicate that activation of the targeted sensor in vivo requires MMP-12. In addition, our in vitro data showed that the enzyme sensor was activated by different MMPs, not only by MMP-12 (Supplemental Figure 5C). Thus, it cannot be excluded that MMPs other than MMP-12 also contribute to the activation of the pan-MMP sensor in wild-type mice, although the lack of activation of the sensor in lungs of MMP-12-deficient mice suggests a dominant role of this proteinase during eosinophil-mediated airway inflammation.

We also investigated MMP activity upon LPS-mediated pulmonary inflammation and compared it with that of our model of OVAinduced allergic airway inflammation described above. For the generation of LPS-mediated pulmonary inflammation, cohorts of 6- to 8-week-old female BALB/c mice received i.n. injection of approximately $15 \mu \mathrm{g}$ LPS in saline (46), while control mice received PBS instead of LPS. All mice were analyzed by FMT either 6,18 , or $24 \mathrm{~h}$ 
A

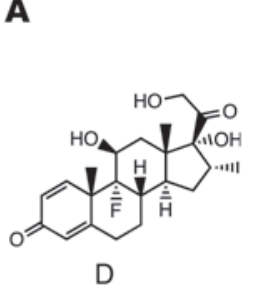

D
B

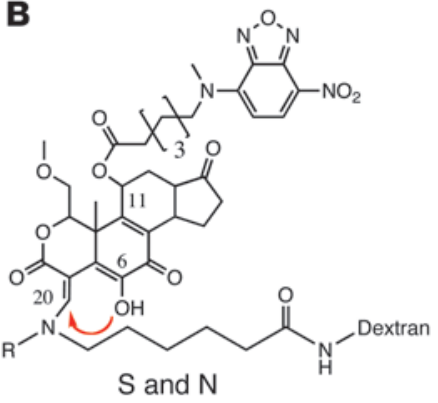

C

D

In vivo MMP activity (FMT)

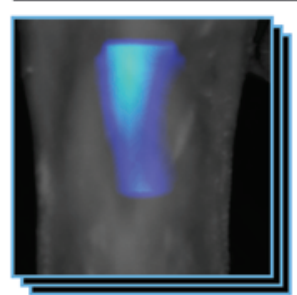

Sens $\times 2$

$\begin{array}{ll}\text { Chall } & - \\ \text { Treat } & \end{array}$

E

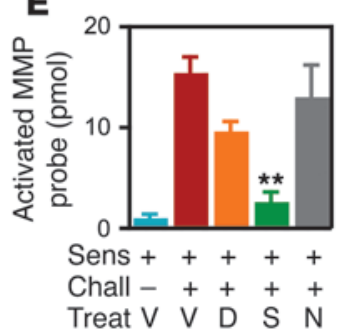

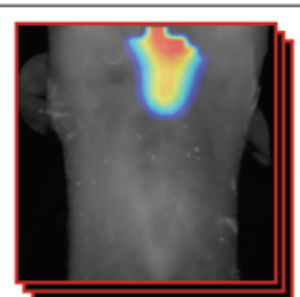

$\times 2$

$\stackrel{+}{V}$

$\mathbf{F}$

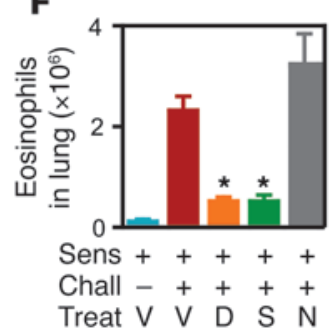

Injection of D, S, N, or V i.v.

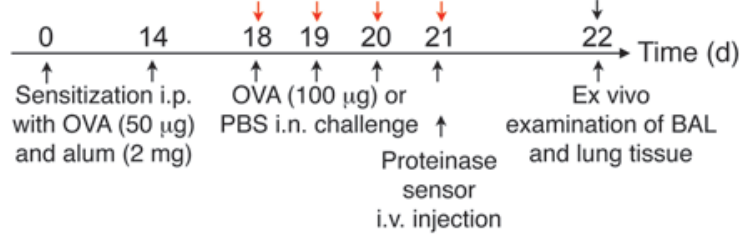

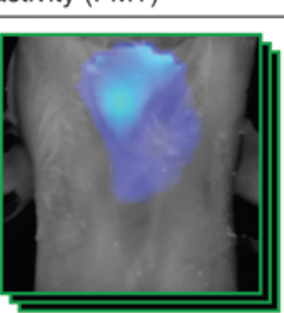

$\times 2$

$\stackrel{+}{\mathrm{S}}$

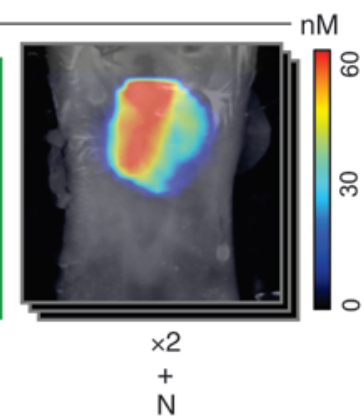

G

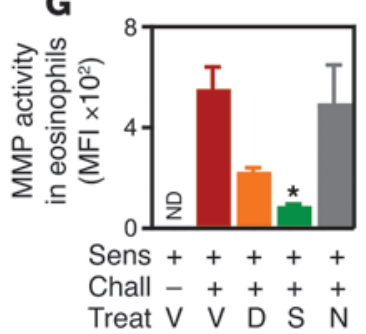

\section{Figure 4}

In vivo FMT for detection of treatment response. (A) Structure of dexamethasone (D). (B) Structures of the self-activating viridin prodrug (S; $R=C H 3)$ and control nonactivating compound $(\mathrm{N} ; \mathrm{R}=\mathrm{H})$. (C) Protocol of procedures and treatment regimen. (D and $\mathbf{E})$ FMT informs on treatment efficacy. Dexamethasone (orange) and self-activating viridin prodrug (green) suppressed eosinophil-associated MMP activity in lung compared with control mice treated with vehicle dextran alone (V; red) or control nonactivating compound (gray). Unchallenged mice (blue) served as controls. (F) Ex vivo analysis of digested lungs. Administration of dexamethasone or self-activating viridin prodrug decreased the number of lung eosinophils. (G) Flow cytometry analysis of digested lungs revealed that administration of self-activating viridin prodrug, and, to a lesser extent, dexamethasone, decreased MMP activity of eosinophils on a per-cell basis. ND, not done. At least 2 independent experiments were performed, with $n=3-5$ per experiment and per group. Data are mean \pm SEM. ${ }^{\star} P<0.05,{ }^{\star \star} P<0.01$ versus OVA-challenged, vehicle dextran-treated control.

after LPS treatment and received the MMP sensor $24 \mathrm{~h}$ before imaging. Mice were sacrificed immediately after imaging, and BAL fluid and lung tissue were harvested and prepared to obtain single-cell suspensions. H\&E staining revealed potent infiltration of immune cells in the BAL of both LPS- and OVA-treated mice. However, these cells were of different types: the LPS treatment recruited mainly neutrophils, whereas the OVA treatment recruited eosinophils, as expected. In fact, we observed a 24:1 neutrophil/eosinophil ratio after LPS treatment and a 1:780 ratio after OVA treatment (Supplemental Figure 6, A and B). Analysis of digested lung tissues revealed similar findings (Supplemental Figure 6C). Importantly, FMT analysis revealed negligible in vivo MMP activity in the lungs of LPS-treated mice compared with those of OVA-treated mice (Supplemental Figure 6D). The near absence of MMP activity in LPS-treated mice was further confirmed ex vivo by FRI on excised lung tissue (data not shown) and by flow cytometry (Supplemental Figure 6E). Thus the MMP sensor is selectively activated in mice with OVA-induced allergic airway inflammation (i.e., by activated eosinophils present in the airways), but not after LPS treatment (i.e., without activated eosinophils). This finding indicates that the sensor selectively reports on eosinophil-mediated MMP activity in vivo.

Noninvasive FMT imaging of treatment response. Having established that FMT can be used as a noninvasive in vivo readout to quantitatively assess eosinophil-mediated lung inflammation, we next used this method to further evaluate the efficacy of therapeutic interventions. First, we tested dexamethasone (Figure 4A and Supplemental Figure 7), a well-established antiinflammatory glucocorticoid that impairs the viability of eosinophils and their recruitment to the lungs in asthmatics as well as in murine models of allergic airway inflammation (47). FMT revealed that administration of the drug to $\mathrm{BALB} / \mathrm{c}$ mice sensitized and challenged with OVA reduced pulmonary MMP activity by about $40 \%$ in vivo (Figure 4 , $\mathrm{D}$ and $\mathrm{E}$ ). Ex vivo analysis also indicated reduced numbers of lung eosinophils (Figure 4F) and decreased MMP activity per eosino- 
A

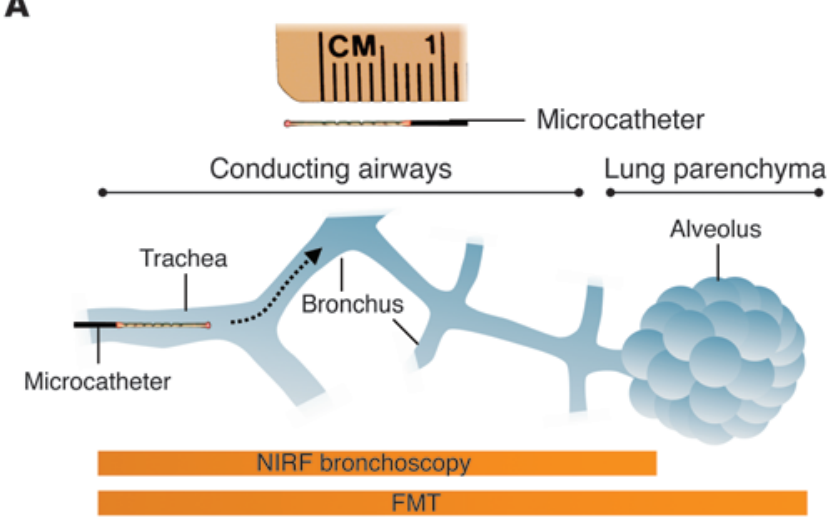

B

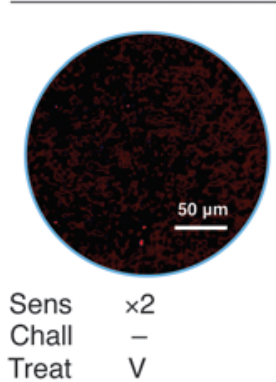

In vivo MMP activity (NIRF bronchoscopy)

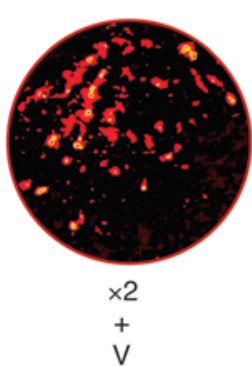

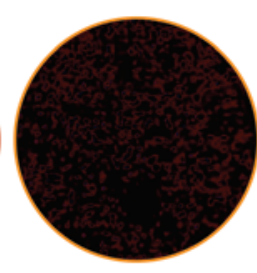

$\times 2$

$+$

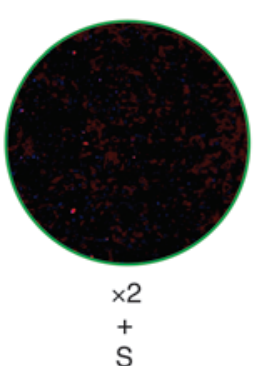

C

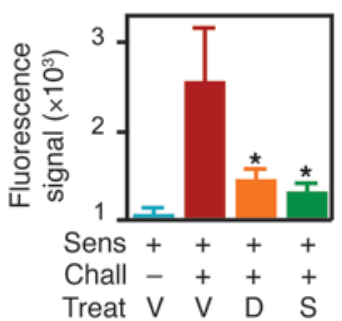

D

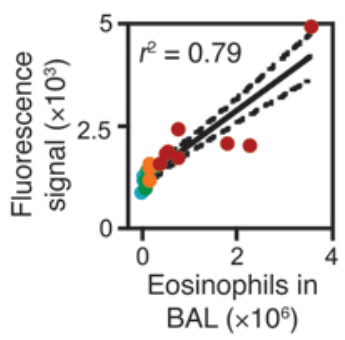

Figure 5

In vivo NIRF bronchoscopy for detection of treatment response. (A) Schematic representation of the airways and features of imaging modalities. FMT resolves signals coming from the entire organ, whereas NIRF fiberoptic bronchoscopy resolves signals coming mostly from the conducting airways. Imaging resolutions are $1 \mathrm{~mm}$ and $15 \mu \mathrm{m}$ for FMT and bronchoscopy, respectively. (B and C) Microcatheter-based NIRF bronchoscopy quantitatively informed on MMP activity within bronchi of mice sensitized and challenged with OVA. (B) Third-order branches are shown. Scale bar: $50 \mathrm{um}$. (C) The signal was dramatically reduced in mice administered dexamethasone (orange) or the self-activating viridin prodrug (green) compared with mice administered vehicle dextran only (red). Unchallenged mice (blue) served as controls. (D) In vivo MMP activity detected by NIRF bronchoscopy positively correlated with the number of eosinophils in BAL fluids $\left(r^{2}=0.79\right)$. At least 2 independent experiments were performed, with $n=3-5$ per experiment and per group. Data are mean \pm SEM. ${ }^{*} P<0.05$ versus OVA-challenged, vehicle dextran-treated control.

phil (Figure 4G) in treated mice. In fact, MMP activity detected by FMT positively correlated with the number of eosinophils in airways $\left(r^{2}=0.71\right)$. Thus, we concluded that FMT allows noninvasive and quantitative assessment of treatment efficacy.

Second, we tested a self-activating viridin prodrug (Figure 4B and Supplemental Figure 7) that uses NBD-wortmannin as viridin. The self-activating viridin prodrug undergoes slow self activation to release low and sustained levels of NBD-wortmannin $(48,49)$, increasing its potency. Wortmannin is a potent inhibitor of PI3K and other kinases (50-53). Therefore, in light of the beneficial effects of PI3K inhibitors in models of inflammatory disorders (54), we tested the self-activating viridin prodrug directly in vivo, as we had done with dexamethasone. We also included 2 controls, a nonactivating viridin that does not release NBD-wortmannin and vehicle dextran alone (Figure 4C). FMT revealed that administration of the self-activating viridin prodrug reduced pulmonary MMP activity by approximately $85 \%$ (Figure 4, D and E), which was confirmed by ex vivo analysis (Figure 4, F and G). Thus, FMT-based molecular imaging represents a method of choice in preclinical models for rapid in vivo testing of new drugs. The precise molecular mechanism responsible for the antiinflammatory activity of the self-activating viridin prodrug remains to be defined, although our data indicate that it can decrease both the accumulation and the enzyme activity (on a per-cell basis) of eosinophils in inflamed lungs.
NIRF fiberoptic bronchoscopy to assess treatment response. In parallel to imaging inflamed lung parenchyma by FMT, we used videoassisted microcatheter NIRF bronchoscopy for direct visualization of NIRF signal in mouse conducting airways (Figure $5 \mathrm{~A}$ and Supplemental Video 5). The imaging microcatheter, a 300- $\mu \mathrm{m}$ outer diameter fiber connected to a laser scanning collection unit, could be atraumatically advanced through third-order branches within mouse bronchi, with visualization of fourth-order branches. We used this imaging modality to interrogate eosinophil-associated MMP activity in real time and at cellular resolution in mice with allergic airway inflammation and upon therapeutic intervention. Video recording of third-order branches revealed potent activity in mice with inflamed airways that received the MMP-targeted sensor (Figure 5, B and C, and Supplemental Videos 6-9). As identified previously in lung parenchyma by IVM, MMP activity in bronchi originated mostly from distinct locations featuring the size and shape of single cells. NIRF bronchoscopy also revealed dramatic reduction of MMP activity in mice treated with dexamethasone or the self-activating viridin prodrug (Figure 5, B and C). MMP activity detected by NIRF bronchoscopy positively correlated with the number of eosinophils (Figure 5D) and with the concentration of IL-5 (data not shown) in BAL fluids. These results indicate the potential of microcatheter NIRF bronchoscopy for noninvasive and quantitative assessment of allergic airway 
inflammation and treatment efficacy. The approach is suited for preclinical and clinical studies.

\section{Discussion}

Enzyme-based activatable sensors for fluorescence-mediated imaging have proven valuable for the detection of protease activity in cancer, in cardiovascular disease, and in bacteria-derived, LPSmediated inflammation $(40,46,55)$, but their usefulness has not yet been evaluated in allergic airway inflammation and asthma. Here we report that a pan-MMP sensor informed noninvasively on extent and quality of allergic airway inflammation in a classical mouse model. Eosinophil-associated MMP activity was detected by flow cytometry and visualized by IVM, FMT, and NIRF fiberoptic bronchoscopy. The technique is remarkably sensitive, and signals reliably associated with MMP activity.

A wealth of data implicates proteinases such as MMPs in asthma and chronic obstructive pulmonary disease (COPD), but comparatively little is known about the source of these enzymes and precisely how they contribute to allergic airway inflammation (24-29, 33-36). Their presence in the airway during allergen challenge suggests an important role in the acute inflammatory response, while their capacity to cleave preferred peptide sequences renders them an attractive imaging target and putative functional biomarkers for disease. With the help of activatable sensors that report on activity, rather than expression, of a culprit enzyme $(56,57)$, we showed that eosinophils, long known to mediate important and deleterious functions in airway and asthma pathophysiology, were a dominant source of airway pan-MMP. We chose a pan-MMP sensor for imaging of enzyme burden because the disease mobilizes multiple MMPs, and related molecules are being introduced clinically. Results in knockout mice further identified MMP-12 as a dominant contributor to this activity, a finding that is in line with recent comprehensive systems-level approaches (43). The sensor also offers the advantage of in vivo detection by virtue of its wholebody distribution, NIRF, and substrate-dependent activation. Thus, the approach allows for real-time, multiscale imaging in deep tissue without relying on invasive ex vivo manipulation (58). Future studies will need to determine whether the technique is also applicable to screening for protease activity in airway pathologies such as COPD. Such insight will begin to define proteinase regulation and activity across inflammatory conditions in which other cell types dominate.

Imaging of proteinase-associated allergic airway inflammation entails several attractive possibilities for clinical translatability. The results of our studies with dexamethasone and the viridin prodrug attest to the exquisite sensitivities of these technologies in discriminating between different levels of inflammatory burden and suggest a role in screening for treatment efficacy. The method may also be used for diagnosis: by targeting inflammation, it may be used to test for allergic airway disease before the onset of acute attacks. Fiberoptic bronchoscopy is a promising approach for this goal. Indeed, the fiberoptic catheter is applicable to humans, is handheld, and does not require large imaging equipment. It is capable of visualizing protease activity deep in fourth-order bronchi, and a clinical version of the imaging system would be relatively inexpensive. Furthermore, the combined use of the MMP sensor and fiberoptic catheter noninvasively informs on the extent of protease activity in vivo and correlates with the extent of total lung inflammation, as assessed by ex vivo methods. At present, FMT is more suited for experimental research on relatively small animal models. However, one possibility for clinical translation would be adaptation of the finger-pulse oximetry technology (59, 60 ) for tomographic quantification of inflammation in sampled regions of the airway. This would require taping of the device intercostally for localized measurements. Future work will answer whether these or other optical or nonoptical imaging approaches can further improve our ability to diagnose the disease, asses treatment efficacy, and allow clinical translatability. For example, the development of sensors for molecular species that are unique to the eosinophil, and that allow for molecular imaging, would also be useful to study allergic responses.

Advances in molecular imaging have allowed the investigation of biological processes in vivo, noninvasively, and at various scales. Increasing evidence suggests that context-based approaches are necessary, because ex vivo and in vitro techniques fail to recapitulate the complex environmental stimuli that orchestrate a specific response $(23,40)$. Many of these approaches may now be translated to address key biological issues in asthma pathophysiology. As it has occurred in other diseases, molecular imaging will likely facilitate a broad, systems-based understanding of cellular and molecular events that trigger and propagate the disease.

\section{Methods}

Mice. BALB/c and C57BL/6 mice were purchased from Taconic Farms Inc. MMP-12-deficient mice on a C57BL/6 background were a gift from S.D. Shapiro (University of Pittsburgh, Pittsburgh, Pennsylvania, USA). Five days prior to imaging, mice were placed on a highly purified reduced-manganese diet (Harlan) to reduce autofluorescence caused by normal mouse chow diet when imaging. All animal experiments were approved by the Massachusetts General Hospital Subcommittee on Research Animal Care.

Induction of allergic airway inflammation. Groups of mice 8-10 weeks old were sensitized once (on day 0 ) or twice (on days 0 and 14) by i.p. injection of $50 \mu \mathrm{g}$ OVA Grade VII (Sigma-Aldrich) emulsified in $2 \mathrm{mg}$ alum (Sigma-Aldrich) in a total volume of $500 \mu \mathrm{l}$ saline. Mice received 4 i.n. challenges with OVA $(100 \mu \mathrm{g}$ in $30 \mu \mathrm{l}$ saline) on days $8,9,10$, and 11 if sensitized once and on days 18,19 , 20 , and 21 if sensitized twice. Mice underwent noninvasive imaging (e.g., FMT, NIRF bronchoscopy, and IVM) $24 \mathrm{~h}$ after the last challenge. Thereafter, $\mathrm{BAL}$ and lungs were harvested for further ex vivo analysis. Control mice were sensitized with OVA and alum, but received PBS during challenge.

Induction of LPS-mediated pulmonary inflammation. Groups of BALB/c mice 8-10 weeks old received i.n. administration of a single dose of approximately $15 \mu \mathrm{g}$ LPS (Sigma-Aldrich) dissolved in $15 \mu \mathrm{l}$ saline. Mice underwent noninvasive FMT imaging 6, 18, and $24 \mathrm{~h}$ after LPS administration. Thereafter, BAL and lungs were harvested for further ex vivo analysis. Control mice received PBS instead of LPS.

Cell recovery. For recovery of cells from the in airway lumen, the trachea was isolated by blunt dissection, and small-caliber tubing was inserted and secured in the airway. Three volumes of $500 \mu \mathrm{l}$ PBS with $0.1 \%$ BSA were instilled, gently aspirated, and pooled. Cells from BAL were pelleted by centrifugation and resuspended in PBS until used. Differential cell counts were performed on BAL cells by counting at least 500 cells on cytocentrifuged preparations (Shandon EZ Single Cytofunnel; Thermo Scientific) stained with H\&E (Protocol HEMA 3; Fisher Scientific) according to the manufacturer's protocol. Cells were differentiated by standard hematologic procedures based on cell morphology. BAL supernatants were kept at $-70^{\circ} \mathrm{C}$ until used for the determination of IL-5 cytokine levels. To recover cells from within the lung parenchyma, lungs were perfused abundantly with PBS, immediately analyzed by FRI, and cut into pieces and incubated in $10 \mathrm{ml}$ RPMI 1640 (Gibco; Invitrogen) containing $0.2 \mathrm{mg} / \mathrm{ml}$ collagenase type IV (Sigma-Aldrich) for $1 \mathrm{~h}$ at $37^{\circ} \mathrm{C}$. Digested lungs were filtered 
through a sterile $40-\mu \mathrm{m}$ porous nylon mesh, and cells were washed and resuspended in $1 \mathrm{ml}$ PBS with $0.1 \%$ BSA.

Synthesis of self-activating viridin prodrug. The complete syntheses and characterizations of the self-activating viridin prodrug and the nonactivating viridin have been described previously (61). Briefly, the viridin wortmannin (Wm) was reacted at C11 to obtain NBD-Wm (62). The NBD-Wm was then reacted with either $\mathrm{N}(\mathrm{Me})$ hexanoic acid or $\mathrm{N}(\mathrm{H})$ hexanoic acid, and the carboxylic acid was converted to an $\mathrm{N}$-hydroxysuccinide ester (48). The N-hydroxysuccinimide ester of each was reacted with a 70-kDa amino dextran (Invitrogen). The self-activating viridin prodrug and the control nonactivating compound are identical except for a methyl group (Figure 4B) that controls viridin release.

Therapeutic intervention. For treatment response studies, groups of mice sensitized on days 0 and 14 were administered daily and before challenge procedure (days 18, 19, 20, and 21) with i.p. injection of $1 \mathrm{mg} / \mathrm{kg}$ dexamethasone (Sigma-Aldrich) in $100 \mu \mathrm{l}$ saline or with i.v. injection of $0.75 \mathrm{mg} / \mathrm{kg}$ self-activating viridin prodrug in $200 \mu \mathrm{l}$ saline. Mice used as controls received i.v. injection of $0.75 \mathrm{mg} / \mathrm{kg}$ nonactive viridin or $0.75 \mathrm{mg} / \mathrm{kg}$ dextran (all in $200 \mu \mathrm{l}$ saline) or were challenged with PBS. Mice were imaged 18-24 h after the last i.n. challenge, and BAL and lungs were immediately obtained for analysis ex vivo.

Cytokine analysis. Determination of IL-5 in BAL fluid was achieved by standard ELISA using the Quantikine Immunoassay (R\&D Systems) according to the manufacturer's protocol.

$m A$ s and flow cytometry. The following antibodies were used: APC-conjugated anti-CCR3 (R\&D Systems), FITC-conjugated anti-CCR3 (R\&D Systems), PE-conjugated anti-CD90 (clone 53-2.1; BD Biosciences), PE-conjugated anti-B220 (clone RA3-6B2; BD Biosciences), PE-conjugated anti-CD49b (clone DX5; BD Biosciences), PE-conjugated anti-NK1.1 (clone PK136; BD Biosciences), PE-conjugated anti-Ly-6G (clone 1A8; BD Biosciences), and APC-conjugated anti-CD11b (clone M1/70; BD Biosciences). The number of eosinophils, monocytes/macrophages, neutrophils, and other cells was defined as the total number of cells per lung multiplied by the percentage of each cell type identified by flow cytometry (LSRII; BD Biosciences).

IVM. A prototype multichannel upright laser-scanning fluorescent microscope (IV100; Olympus) for intravital observations was used (41). Images were acquired with Fluoview imaging software (FV300, version 4.3; Olympus). Lasers used for excitation included a 10-mW, 633-nm helium-neon and a 18-mW, 748-nm diode laser. Emission signal was filtered through a SDM 570-nm and SDM 750-nm beam splitter and by using 650to 700-nm barrier and 770-nm bandpass filters, respectively.

FMT. Mice received $5 \mathrm{nmol}$ MMPSense-680 or ProSense-750 (both from VisEn Medical) via a tail vein injection. The probes are based on polymeric scaffolds that can be cleaved by target protease, resulting in the release of fluorochromes and extensive fluorescence generation (dequenching). MMPSense-680 excitation and emission wavelengths were $680 \pm 10 \mathrm{~nm}$ and $700 \pm 10 \mathrm{~nm}$, respectively. ProSense-750 excitation and emission wavelengths were $750 \pm 10 \mathrm{~nm}$ and $780 \pm 10 \mathrm{~nm}$. These sensors have a reported blood half-life of about $24 \mathrm{~h}$ and are mostly cleared through the liver (46, 63 ). Mice were anesthetized by inhalation of isoflurane ( $2 \%$ isoflurane, $2 \mathrm{l} /$ min oxygen) $24 \mathrm{~h}$ after administration of the probes, and hair from the thorax and abdomen was removed by shaving and chemical depilation. Mice were immersed in an index-matching fluid to simplify theoretical constrains associated with boundary modeling and to calculate light prop- agation and were imaged using a commercially available FMT imaging system (FMT; VisEn Medical). Quantitative reconstruction of 3-dimensional maps of fluorochromes was based on Born-forward algorithms (64), and control mice not sensitized and not challenged with OVA served for signal normalization. To improve signal visualization and anatomic localization, several mice were also imaged by FMT-CT fusion imaging (64).

FRI. Excised lungs were imaged by FRI (BonSAI; Siemens) using filters for detection of fluorescence at 700 and $750 \mathrm{~nm}$. Data were analyzed using OsiriX and expressed as mean fluorescent counts per second.

NIRF fiberoptic bronchoscopy and image analysis. NIRF bronchoscopy was performed with an intravital fiberoptic confocal fluorescence microscopy system (Cellvizio Lab-660; Mauna Kea Technologies) that consists of a laser scanning collection unit ( $660 \mathrm{~nm}$ excitation; 680-900 nm collection wavelengths) and a S300 fibered microcatheter (bundle of 6,000 imaging fibers contained within a 300- $\mu \mathrm{m}$ diameter). The catheter has an axial resolution of $15 \mu \mathrm{m}$, a lateral resolution of $3.5 \mu \mathrm{m}$, and a field of view of $300 \mu \mathrm{m}$. Mice were administered a gas anesthetic agent ( $2 \%$ isoflurane, $2 \mathrm{l} / \mathrm{min}$ oxygen) before and throughout the imaging procedure. A laryngoscope was used to aid visualization of the mouse vocal cords. The imaging catheter was placed inside a 20 -gauge sheath before insertion into the trachea. This allowed the release of the 20-gauge sheath under direct visualization inside the main bronchus. After removal of the catheter, the sheath was connected to a mechanical ventilator (Inspira ASV; Harvard Apparatus), and data were acquired on a continuous video sequence (12-bit near-infrared images at 12 frames per second). Large portions of the conducting airways were inspected down to third-order branches. Data were acquired and analyzed with ImageCell software (version 3.6.1; Mauna Kea Technologies). The maximum near-infrared signal observed within each frame was averaged across a large set of representative frames acquired (minimum 100) to comprise the fluorescence reading for each mouse bronchoscopy.

Statistics. Data ( $n=3-14$ mice per group) are expressed as mean \pm SEM. Statistical significance was determined by 1 -way ANOVA. Differences between groups were determined by multiple comparisons of the means by using Tukey's multiple comparison test. Significance was accepted for $P$ values less than 0.05 .

\section{Acknowledgments}

This work was supported in part by the Strategic Program for Asthma Research (SPAR) and by NIH grant U24 CA092782. The authors thank S.D. Shapiro for providing MMP-12-deficient mice and Cedric Berger and Sheena Hembrador for imaging.

Received for publication May 28, 2008, and accepted in revised form September 24, 2008.

Address correspondence to: Mikael J. Pittet or Ralph Weissleder, Center for Systems Biology, Massachusetts General Hospital, Harvard Medical School, Simches Research Building, 185 Cambridge St., Boston, Massachusetts 02114, USA. Phone: (617) 7266481; Fax: (617) 726-5708; E-mail: mpittet@mgh.harvard.edu (M.J. Pittet); rweissleder@mgh.harvard.edu (R. Weissleder).

Filip K. Swirski and Peter Waterman contributed equally to this work.
1. Larche, M., Akdis, C.A., and Valenta, R. 2006. Immunological mechanisms of allergen-specific immunotherapy. Nat. Rev. Immunol. 6:761-771.

2. Holgate, S.T., and Polosa, R. 2006. The mechanisms, diagnosis, and management of severe asthma in adults. Lancet. 368:780-793.

3. Barnes, P.J. 2008. Immunology of asthma and chronic obstructive pulmonary disease. Nat. Rev. Immunol. 8:183-192.

4. Noble, P.W., and Jiang, D. 2006. Matrix regulation of lung injury, inflammation, and repair: the role of innate immunity. Proc. Am. Thorac. Soc. 3:401-404.

5. Garcia-Zepeda, E.A., et al. 1996. Human eotaxin is a specific chemoattractant for eosinophil cells and provides a new mechanism to explain tissue eosinophilia. Nat. Med. 2:449-456.

6. Ponath, P.D., et al. 1996. Cloning of the human eosinophil chemoattractant, eotaxin. Expression, receptor binding, and functional properties suggest a mechanism for the selective recruitment of eosinophils. J. Clin. Invest. 97:604-612. 
7. Drazen, J.M., Israel, E., and O’Byrne, P.M. 1999 Treatment of asthma with drugs modifying the leukotriene pathway. N. Engl. J. Med. 340:197-206.

8. Rothenberg,M.E., and Hogan,S.P. 2006. Theeosinophil. Annu. Rev. Immunol. 24:147-174.

9. Busse, W.W., and Lemanske, R.F.J. 2001. Asthma. N. Engl. J. Med. 344:350-362.

10. Walsh, E.R., et al. 2008. Strain-specific requirement for eosinophils in the recruitment of $T$ cells to the lung during the development of allergic asthma. J. Exp. Med. 205:1285-1292.

11. Jacobsen, E.A., et al. 2008. Allergic pulmonary inflammation in mice is dependent on eosinophilinduced recruitment of effector T cells. J. Exp. Med. 205:699-710.

12. Wang, H.B., Ghiran, I., Matthaei, K., and Weller, P.F. 2007. Airway eosinophils: allergic inflammation recruited professional antigen-presenting cells. J. Immunol. 179:7585-7592.

13. Huber, H.K., and Koessleder, K.K. 1922. The pathology of bronchial asthma. Arch. Int. Med. 30:689-760.

14. Bousquet, J., et al. 1990. Eosinophilic inflammation in asthma. N. Engl. J. Med. 323:1033-1039.

15. Bradley, B.L., et al. 1991. Eosinophils, T-lymphocytes, mast cells, neutrophils, and macrophages in bronchial biopsy specimens from atopic subjects with asthma: comparison with biopsy specimens from atopic subjects without asthma and normal control subjects and relationship to bronchial hyperresponsiveness. J. Allergy Clin. Immunol. 88:661-674.

16. Robinson, D.S., et al. 1992. Predominant TH2like bronchoalveolar T-lymphocyte population in atopic asthma. N. Engl. J. Med. 326:298-304.

17. Miller,M.R., et al. 2005. Standardisation of spirometry. Eur. Respir. J. 26:319-338.

18. Niimi, A., et al. 2004. Clinical assessment of airway remodeling in asthma: utility of computed tomography. Clin Rev Allergy Immunol. 27:45-58.

19. Samee, S., et al. 2003. Imaging the lungs in asthmatic patients by using hyperpolarized helium-3 magnetic resonance: assessment of response to methacholine and exercise challenge. J. Allergy Clin Immunol. 111:1205-1211.

20. Driehuys, B., et al. 2006. Imaging alveolar-capillary gas transfer using hyperpolarized 129Xe MRI. Proc. Natl. Acad. Sci. U. S. A. 103:18278-18283.

21. Venegas, J.G., et al. 2005. Self-organized patchiness in asthma as a prelude to catastrophic shifts. Nature. 434:777-782.

22. Schuster, D.P. 2007. The opportunities and challenges of developing imaging biomarkers to study lung function and disease. Am. J. Respir. Crit. Care Med. 176:224-230.

23. Pittet, M.J., and Mempel, T.R. 2008. Regulation of T-cell migration and effector functions: insights from in vivo imaging studies. Immunol. Rev. 221:107-129.

24. Pouladi, M.A., et al. 2004. Interleukin-13-dependent expression of matrix metalloproteinase-12 is required for the development of airway eosinophilia in mice. Am. J. Respir. Cell Mol. Biol. 30:84-90.

25. Kelly, E.A., and Jarjour, N.N. 2003. Role of matrix metalloproteinases in asthma. Curr. Opin. Pulm. Med. 9:28-33.

26. Lanone, S., et al. 2002. Overlapping and enzymespecific contributions of matrix metalloproteinases- 9 and -12 in IL-13-induced inflammation and remodeling. J. Clin. Invest. 110:463-474.
27. Cataldo, D.D., et al. 2002. Matrix metalloproteinase- 9 deficiency impairs cellular infiltration and bronchial hyperresponsiveness during allergen-induced airway inflammation. Am. J. Pathol. 161:491-498.

28. Lee, Y.C., et al. 2001. A murine model of toluene diisocyanate-induced asthma can be treated with matrix metalloproteinase inhibitor. J. Allergy Clin. Immunol. 108:1021-1026.

29. Kumagai, K., et al. 1999. Inhibition of matrix metalloproteinases prevents allergen-induced airway inflammation in a murine model of asthma. J. Immunol. 162:4212-4219.

30. Fajardo, I., Svensson, L., Bucht, A., and Pejler, G. 2004. Increased levels of hypoxia-sensitive proteins in allergic airway inflammation. Am. J. Respir. Crit. Care Med. 170:477-484.

31. Zheng, T., et al. 2000. Inducible targeting of IL-13 to the adult lung causes matrix metalloproteinaseand cathepsin-dependent emphysema. J. Clin. Invest. 106:1081-1093.

32. Cimerman, N., et al. 2001. Serum concentration and circadian profiles of cathepsins $\mathrm{B}, \mathrm{H}$ and $\mathrm{L}$, and their inhibitors, stefins A and B, in asthma. Clin. Chim. Acta. 310:113-122.

33. Xie, S., et al. 2005. Induction and regulation of matrix metalloproteinase-12 in human airway smooth muscle cells. Respir Res. 6:148.

34. Mautino, G., Capony, F., Bousquet, J., and Vignola, A.M. 1999. Balance in asthma between matrix metalloproteinases and their inhibitors. J. Allergy Clin. Immunol. 104:530-533.

35. Hoshino, M., Nakamura, Y., Sim, J., Shimojo, J., and Isogai, S. 1998. Bronchial subepithelial fibrosis and expression of matrix metalloproteinase- 9 in asthmatic airway inflammation. J. Allergy Clin. Immunol. 102:783-788

36. Parks, W.C., Wilson, C.L., and Lopez-Boado, Y.S 2004. Matrix metalloproteinases as modulators of inflammation and innate immunity. Nat. Rev. Immunol. 4:617-629.

37. Reed, C.E., and Kita, H. 2004. The role of protease activation of inflammation in allergic respiratory diseases. J. Allergy Clin. Immunol. 114:997-1008; quiz 1009.

38. Gueders, M.M., Foidart, J.M., Noel, A., and Cataldo, D.D. 2006. Matrix metalloproteinases (MMPs) and tissue inhibitors of MMPs in the respiratory tract: potential implications in asthma and other lung diseases. Eur. J. Pharmacol. 533:133-144.

39. Laitinen, L.A., et al. 1996. Bronchial biopsy findings in intermittent or "early" asthma. J. Allergy Clin. Immunol. 98:S3-S6; discussion S33-S40.

40. Weissleder, R., and Pittet, M.J. 2008. Imaging in the era of molecular oncology. Nature 452:580-589.

41. Alencar, H., Mahmood, U., Kawano, Y., Hirata, T., and Weissleder, R. 2005. Novel multiwavelength microscopic scanner for mouse imaging. Neoplasia. 7:977-983

42. Strickland, D.H., et al. 2006. Reversal of airway hyperresponsiveness by induction of airway mucosal CD4+CD25+ regulatory T cells. J. Exp. Med. 203:2649-2660

43. Novershtern, N., Itzhaki, Z., Manor, O., Friedman, N., and Kaminski, N. 2008. A functional and regulatory map of asthma. Am. J. Respir. Cell Mol. Biol. 38:324-336.

44. Cataldo, D.D., et al. 2003. Pathogenic role of matrix metalloproteases and their inhibitors in asthma and chronic obstructive pulmonary disease and therapeu- tic relevance of matrix metalloproteases inhibitors. Cell. Mol. Biol. (Noisy-le-grand). 49:875-884.

45. Demedts, I.K., et al. 2006. Elevated MMP-12 protein levels in induced sputum from patients with COPD. Thorax. 61:196-201.

46. Haller, J., et al. 2008. Visualization of pulmonary inflammation using noninvasive fluorescence molecular imaging. J. Appl. Physiol. 104:795-802.

47. Barnes, P.J., and Pedersen, S. 1993. Efficacy and safety of inhaled corticosteroids in asthma. Report of a workshop held in Eze, France, October 1992. Am. Rev. Respir. Dis. 148:S1-S26.

48. Yuan, H., Luo, J., Weissleder, R., Cantley, L., and Josephson, L. 2006. Wortmannin-C20 conjugates generate wortmannin. J. Med. Chem. 49:740-747.

49. Blois, J., et al. 2008. Slow self-activation enhances the potency of viridin prodrugs. J. Med. Chem. 51:4699-4707.

50. Yano, H., et al. 1993. Inhibition of histamine secretion by wortmannin through the blockade of phosphatidylinositol 3-kinase in RBL-2H3 cells. J. Biol. Chem. 268:25846-25856.

51. Zhu, T., et al. 2006. Pegylated wortmannin and 17hydroxywortmannin conjugates as phosphoinositide 3-kinase inhibitors active in human tumor xenograft models. J. Med. Chem. 49:1373-1378.

52. Sarkaria, J.N., et al. 1998. Inhibition of phosphoinositide 3 -kinase related kinases by the radiosensitizing agent wortmannin. Cancer Res. 58:4375-4382.

53. Wymann, M.P., et al. 1996. Wortmannin inactivates phosphoinositide 3-kinase by covalent modification of Lys-802, a residue involved in the phosphate transfer reaction. Mol. Cell. Biol. 16:1722-1733.

54. Rommel, C., Camps, M., and Ji, H. 2007. PI3K delta and PI3K gamma: partners in crime in inflammation in rheumatoid arthritis and beyond? Nat. Rev. Immunol. 7:191-201.

55. Jaffer, F.A., and Weissleder, R. 2005. Molecular imaging in the clinical arena. JAMA. 293:855-862.

56. Weissleder, R., Tung, C.H., Mahmood, U., and Bogdanov, A.J. 1999. In vivo imaging of tumors with protease-activated near-infrared fluorescent probes. Nat. Biotechnol. 17:375-378.

57. Bremer, C., Tung, C.H., and Weissleder, R. 2001. In vivo molecular target assessment of matrix metalloproteinase inhibition. Nat. Med. 7:743-748.

58. Ntziachristos, V., Tung, C.H., Bremer, C., and Weissleder, R. 2002. Fluorescence molecular tomography resolves protease activity in vivo. Nat. Med. 8:757-760.

59. Severinghaus, J.W., and Kelleher, J.F. 1992. Recent developments in pulse oximetry. Anesthesiology. 76:1018-1038

60. Yelderman, M., and New, W.J. 1983. Evaluation of pulse oximetry. Anesthesiology. 59:349-352.

61. Blois, J., et al. 2008. Slow self-activation enhances the potency of viridin prodrugs. J. Med. Chem. 51:4699-4707.

62. Barnes, K.R., et al. 2008. Fate of a bioactive fluorescent wortmannin derivative in cells. Bioconjug. Chem. 19:130-137.

63. Weissleder, R., Bogdanov, A.J., Tung, C.H., and Weinmann, H.J. 2001. Size optimization of synthetic graft copolymers for in vivo angiogenesis imaging. Bioconjug. Chem. 12:213-219.

64. Grimm, J., et al. 2005. Use of gene expression profiling to direct in vivo molecular imaging of lung cancer. Proc. Natl. Acad. Sci. U. S. A. 102:14404-14409. 